\title{
THE IMPOSSIBILITY OF DESUSPENDING COLLAPSES
}

\author{
BY W. B. R, LICKORISH AND J, M. MARTIN ${ }^{1}$
}

Communicated by R. H. Bing, April 8, 1968

It is known that in order to prove the polyhedral Schoenflies conjecture in all dimensions, it is enough to show that, if $\left(B^{4}, B^{3}\right)$ is a $(4,3)$ ball pair, then $B^{4}$ collapses (polyhedrally) to $B^{3}$. Recently, using the solution to the polyhedral Poincare conjecture in high dimensions, Husch has shown [3] that if $\left(B^{7}, B^{6}\right)$ is a $(7,6)$ ball pair, then $B^{7}$ collapses to $B^{6}$. It is tempting to try to prove that $B^{4}$ collapses to $B^{3}$ by invoking the following conjecture.

Conjecture A. If $M$ is a polyhedral manifold, $L$ a submanifold of $M$ and $S(M) \backslash S(L)$, then $M \searrow L .(S(X)$ denotes the suspension of $X$ and " \" denotes a polyhedral collapse.)

If Conjecture $A$ were true we could suspend a $(4,3)$ ball pair three times to obtain a $(7,6)$ ball pair, use Husch's result, and then apply Conjecture A three times in order to desuspend the collapse.

In this note we present a counterexample to Conjecture $A$, and discuss other conjectures related to the problem of desuspending collapses.

Example 1. Let $M^{4}$ be a polyhedral 4-manifold, as described in [4] or [5], with the following properties. (a) $M^{4}$ is contractible, (b) $\pi_{1}(\partial M) \neq 0$, (c) $M^{4} \times I \cong B^{5}$. Consider $S\left(M^{4}\right)$ as $M^{4} \times I$ together with a cone on $M^{4} \times\{0\}$ and another cone on $M^{4} \times\{1\}$. Thus if $v_{0}$ and $v_{1}$ are the vertices of these cones,

$$
S\left(M^{4}\right)=\left(M^{4} \times I\right) \cup\left(v_{0} *\left(M^{4} \times\{0\}\right)\right) \cup\left(v_{1} *\left(M^{4} \times\{1\}\right)\right) .
$$

Now let $B^{3}$ be a 3 -ball in $\partial M^{4}$. Since $M^{4} \times I$ is a 5-ball, with $B^{3} \times I$ as a face, there is an elementary collapse

$$
M^{4} \times I \searrow\left(M^{4} \times\{0\}\right) \cup\left(M^{4} \times\{1\}\right) \cup\left[\left(\partial M^{4}-\operatorname{int} B^{3}\right) \times I\right],
$$

Thus there is a collapse

$$
S\left(M^{4}\right) \searrow\left(v_{0} *\left(M^{4} \times\{0\}\right)\right) \cup\left(v_{1} *\left(M^{4} \times\{1\}\right)\right) \cup\left(\left(\partial M^{4}-\operatorname{int} B^{3}\right) \times \mathrm{I}\right),
$$

Now, by collapsing conewise $v_{i} *\left(M^{4} \times\{i\}\right)$ to $v_{i} *\left(\left(\partial M^{4}-\operatorname{int} B^{3}\right)\right.$. $\times\{i\})$, for $i=0$ and 1 , we have $S\left(M^{4}\right) \backslash S\left(\partial M^{4}-\operatorname{int} B^{3}\right)$. However, since $\pi_{1}\left(M^{4}\right)=0$ and $\pi_{1}\left(\partial M^{4}-\operatorname{int} B^{3}\right) \neq 0, M^{4} \times \partial M^{4}-\operatorname{int} B^{3}$. This provides a counter-example to Conjecture $A$.

REMARK 1. By taking two copies of the above manifold, $M_{1}$ and

1 This paper was written while the second author was a fellow of the Alfred P. Sloan Foundation. 
$M_{2}, 3$-balls $B_{1}$ and $B_{2}$ in their boundaries and identifying $\partial M_{1}-\operatorname{int} B_{1}$ with $\partial M_{2}-$ int $B_{2}$, one can obtain a similar counter-example in which $M=M_{1} \cup M_{2}$ is a 4 -ball, and $L=\partial M_{1}-\operatorname{int} B_{1}$ is properly imbedded in $M$.

REMARK 2. By adapting Example 1, the following can be proved. There exists a polyhedron $X$ and a point $x \in X$ such that $S(X) \backslash S(x)$ but $X$ is not collapsible. Take $X$ as $X=M^{4} \cup\left(x *\left(\partial M^{4}-\operatorname{int} B^{3}\right)\right)$; i.e. $X$ is the 4-manifold mentioned in Example 1 together with a cone on its boundary less the interior of a 3-ball. Now $S(X) \backslash S(x)$ by a similar argument to that used above. Suppose $X$ is collapsible. Then $X \searrow x$ (as a collapsible polyhedron collapses to any given point). Then $X$ is P.L. homeomorphic to a regular neighbourhood of $x$ in $X$ (regular neighbourhoods in polyhedra are defined and extensively discussed in [2]). $x *\left(\partial M^{4}-\operatorname{int} B^{3}\right)$ is such a regular neighbourhood, so by the regular neighbourhood uniqueness theorem [2] there is a P.L. homeomorphism

$$
h: X, x \rightarrow x *\left(\partial M^{4}-\operatorname{int} B^{3}\right), x .
$$

Hence restricting $h$ to the points of $X$ which do not have neighbourhoods which are open 4-cells, $h$ maps the 3 -sphere $B^{3} \cup\left(x * \partial B^{8}\right)$ homeomorphically onto $\left(\partial M^{4}-\operatorname{int} B^{3}\right) \cup\left(x * \partial B^{3}\right)$ which is homeomorphic to $\partial M^{4}$. This is impossible as $\pi_{1}\left(\partial M^{4}\right) \neq 0$, and hence $X$, is not collapsible.

We now turn our attention to a problem involving simplicial collapsing. Bing [1] has given an example of a triangulation of a 3-cell which is not collapsible. One would hope to be able to suspend this triangulation to obtain noncollapsible triangulations of the $n$-cell. This leads to Conjecture B.

*Conjecture B. If $K$ is a complex, $L$ is a subcomplex of $K$, and $S(K) \searrow S(L)$, then $K \searrow^{s} L$. ("\" denotes simplicial collapsing.)

We do not know the answer to Conjecture B, but it seems likely that it is false (although it is not difficult to prove it true if $K$ is only two-dimensional). The following question is related to Conjecture $\mathrm{B}$.

Qunstion 1. Is there a complex $K$, with subcomplexes $X$ and $Y$ such that $K \searrow X, K \searrow Y, K \searrow X \cup Y$, but $K \dot{\otimes}: X \cap Y$ ?

An affirmative answer to Question 1 would provide a counterexample to Conjecture B as follows: Suppose that $K, X$ and $Y$ have the properties stated in Question 1. Consider $S(K)$ as $(a \cup b) * K$ where $a$ and $b$ are two points. Now since $K \searrow X, S(K) \searrow(a * X)$ $\cup(b * K)$. Since $K \unlhd Y, \quad(a * X) \cup(b * K) \unlhd(a * X) \cup K \cup(b * Y)$. This latter complex collapses simplicially to $(a * X) \cup(b * Y)$ because

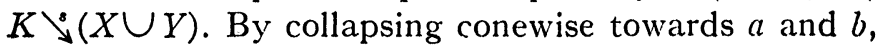

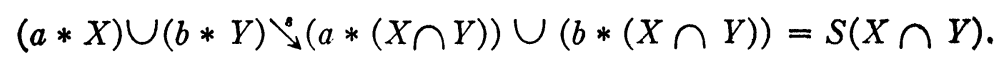


Thus

$$
S(K) \searrow S(X \cap Y) \text { but } K \dot{X} X \cap Y \text {. }
$$

Using the manifold $M^{4}$ employed in Example 1, it is possible to show as follows that Question 1 would have an affirmative answer if polyhedral collapsing replaced simplicial collapsing.

EXAMPLE 2. Let $M^{4}$ be the manifold used in Example 1, and let $B^{3}$ be a 3 -ball in $\partial M^{4}$ as before. Let $X$ and $Y$ be sub-polyhedra of $M^{4} \times I$ defined by

$$
\begin{aligned}
& X=\left(M^{4} \times\{0\}\right) \cup\left(\left(\partial M^{4}-\operatorname{int} B^{3}\right) \times I\right) \\
& Y=\left(M^{4} \times\{1\}\right) \cup\left(\left(\partial M^{4}-\operatorname{int} B^{3}\right) \times I\right) .
\end{aligned}
$$

Using the product structure of $M^{4} \times I$,

$$
M^{4} \times I \searrow X \text { and } M^{4} \times I \searrow Y \text {. }
$$

Because $M^{4} \times I$ is a 5 -ball, $M^{4} \times I \searrow X \cup Y$, but $M^{4} \times I \times X \cap Y$ since $X \cap Y=\left(\left(\partial M^{4}-\operatorname{int} B^{3}\right) \times I\right)$ is not simply connected.

Question 2. With $M^{4}, X$ and $Y$ as in Example 2, is there a triangulation of $M^{4} \times I$, triangulating $X$ and $Y$ as subcomplexes, so that $M^{4} \times I \searrow X, M^{4} \times I \searrow Y$, and $M^{4} \times I \searrow^{*} X \cup Y$ ?

* Added in proof. The answer to Question 2 is "Yes." L. C. Glaser has pointed out that this follows at once from Theorem 7 of J. H. C. Whitehead, Simplicial spaces, nuclei and m-groups, Proc. London Math Soc. 45 (1939), 243-327. Thus Question 1 has an affirmative answer, and so Conjecture $B$ is false.

\section{REFERENCES}

1. R. H. Bing, Some aspects of the topology of 3-manifolds related to the Poincare conjecture, Lectures on modern mathematics, Vol. II, Wiley, New York, 1964.

2. M. M. Cohen, $A$ general theory of relative regular neighborhoods (to appear).

3. L. S. Husch, On collapsible ball pairs (to appear).

4. B. Mazur, A note on some contractible 4-manifolds, Ann. Math. 73 (1961), 221-228.

5. V. Poénaru, La décomposition de l'hypercube en produit topologique, Bull. Soc. Math. France 88 (1960), 113-129.

UNIVERSITY OF Wisconsin, MADISON 